\title{
A Reduced-Complexity Mixer Linearization Scheme
}

\author{
Paul A. Warr and Alan M. Potter \\ Centre for Communications Research, University of Bristol, Bristol BS8 1UB, UK \\ Correspondence should be addressed to Paul A. Warr, paul.a.warr@bristol.ac.uk
}

Received 20 May 2009; Accepted 22 July 2009

Recommended by Nikos Sagias

Measurement results of the signals emanating from both IF and LO ports of a double balanced mixer are presented, and, thus, it is shown that the linearization of the output in a down-converting mixer by the summation of the IF signal and the signal emanating from the LO or RF port is feasible. Feedforward-based architectures for the linearization of down-conversion mixers are introduced that exploit this phenomenon, and linearity performance results of the frequency translation of both two-tone and TETRA-modulated signals are presented. This technique employs only a single mixer and hence overcomes the complexity of other mixer linearization schemes. The overall processing gain of the system is limited by the level of wanted signal present in the error signal.

Copyright ( 2009 P. A. Warr and A. M. Potter. This is an open access article distributed under the Creative Commons Attribution License, which permits unrestricted use, distribution, and reproduction in any medium, provided the original work is properly cited.

\section{Introduction}

Intermodulation Distortion (IMD), caused by odd-order nonlinearities, falls close to the required signal and is impracticable to remove by filtering. Of most concern with double-balanced diode mixers is third-order intermodulation distortion (3IMD) as this is the greatest contributor to the reduction in system dynamic range. Normally, if this would be a problem within a system, a higher LO power mixer would be chosen to increase the linearity of the process. Alternatively, these in-band IMD products may be suppressed using a linearization method.

In standard techniques for the linearization of mixers $[1,2]$ a second frequency translation step is required to ensure that the signal to be linearized and the error signal are both at the same frequency. Thus, a second mixer is required which both increases complexity and limits performance. However, in the technique presented here, the fact that mixers output distortion at the desired output frequency at all ports is exploited such that a second mixer is not required. Therefore, a greatly simplified architecture may be used comprising a single balanced suppression loop rather than two, as commonly proposed.

This paper describes a topology for the linearization of a mixer, used within the down-conversion process of a radio receiver. This topology is developed from an experimental analysis of the linear and nonlinear characteristics of a double balanced diode mixer. In [3] a scheme for linearizing amplifiers using backward-traveling signals is proposed. The experimental analysis reported here shows that a similar technique may be applied to mixers.

At the system level, the mixer is a generator of distortion (i.e., it may be considered as the source of the undesired nonlinear output), and so the balance of the distortion energy observed at the three ports is not the same as that of the wanted signal. This leads to different signal-to-distortion levels of the IF signal appearing at the IF, LO, and RF ports and, thus, a mixer linearization scheme without the use of a second mixer is feasible.

By gain and phase adjustment of the IF signal found at the LO or RF ports of the mixer it is possible to create an error signal which can be fed forward to suppress the distortion in the output IF signal [4]. In this paper, as an example, the IF signal emanating from the LO port is used to increase the signal-to-distortion ratio at the IF output of the network.

There are two performance limitations in this approach; one is due to the fidelity of the linearization information (i.e., the amount of residual wanted signal power in the error signal), and the other is due to the presence of noise in the error signal. The resulting error signal may be considered as inherent as it is neither formed by a suppression loop architecture for the removal of wanted 


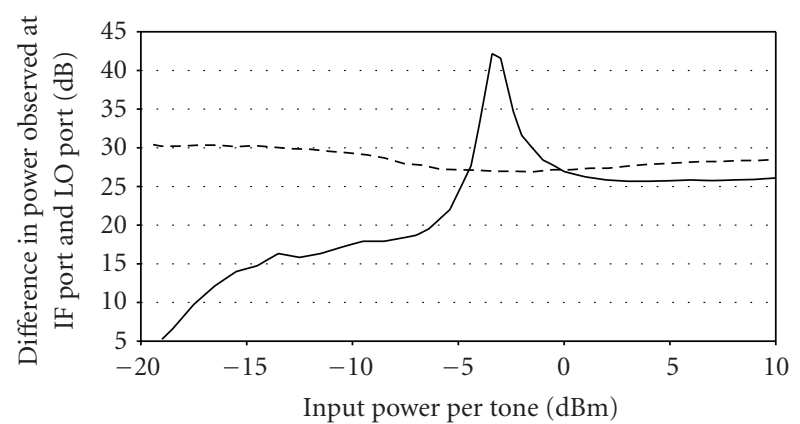

FIGURE 1: Difference between both 3IMD and wanted signal power at LO port and IF port, with input power $(900 \mathrm{MHz}$ RF and $200 \mathrm{MHz}$ IF). The hatched trace is the wanted signal difference and the solid trace is the 3IMD difference.

energy, nor formed by the application of a model of the mixer nonlinear performance.

\section{Mixer Output Measurements at IF and LO Ports}

For a standard double-balanced diode mixer, there is a signal at the LO and RF ports at the IF band, and the wantedto-3IMD ratio of this signal is similar to that produced by a second mixer subjected to a higher input power level. Figure 1 shows the power difference between the wanted signal and 3IMD at the IF and LO ports, for a Minicircuits TUF-5sm level 7 mixer [5].

For the linearization scheme to be successful, a high difference across ports in the wanted signal power and low difference in the 3IMD power are desired, such that only the latter is suppressed. Thus, referring to Figure 1, a maximum input power around $-7 \mathrm{dBm}$ per tone would generate a system processing gain that may be exploited. Operation at lower input power would provide additional linearity enhancement. However, this must be traded-off against the overall loss of the process and the noise this will introduce into the system.

For ideal operation of the inherent-signal single-loop feedforward scheme, there should be constant difference with input power of the distortion at the two ports of the mixer. However, Figure 1 shows a considerable variation in this parameter over varying input powers. For the maximum exploitation of the inherent error signal in a linearization scheme, the mixer should be designed from the diode level for a specific application such that the greatest difference in 3IMD coincides with the required operation frequencies ( $R F$ and IF), input power and bandwidth.

\section{Simple Architectures to Exploit the Inherent Error Signal}

The IF signal at either the RF (Figure 2) or LO (Figure 3) port of the mixer may be employed as an error signal; it is first gain-balanced, such that the 3IMD power matches that in the IF output path. By combining these two paths in antiphase,

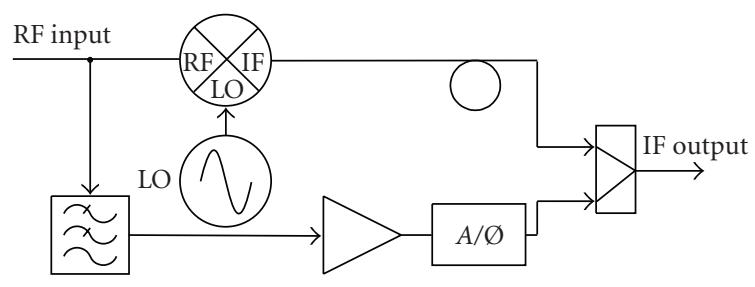

FIGURE 2: Single-loop feedforward linearization topology utilizing the IF distortion present at the RF port.

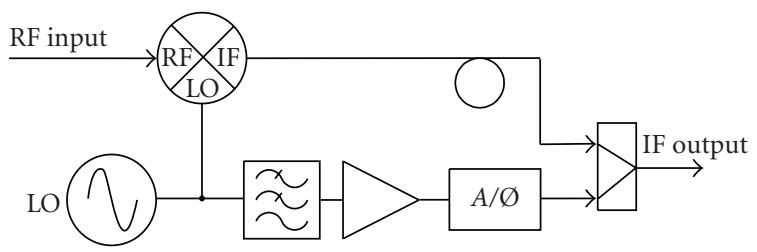

FIGURE 3: Single-loop feedforward linearization topology utilizing the IF distortion present at the LO port.

the distortion is suppressed. The output level of the wanted signal may be reduced due to the presence of wanted signal energy in the error path. However, referring to Figure 1, the maximum suppression of the wanted signal when the 3IMD is power-matched will be $3.3 \mathrm{~dB}$, if the input power per tone is maintained at $-7 \mathrm{dBm}$. It should be noted that this is a worse case suppression as the phase relationship between the wanted signal across ports and the 3IMD across ports is not correlated. Indeed, negligible wanted signal suppression is shown in Section 4.

A filter removes the LO from the signal and it is amplified to the required level to match the IF output of the mixer. The LO filtering requirement is most prevalent in the case of taking the error signal from the LO port (Figure 3). However, in a down-conversion stage the IF and the LO are spectrally far apart, relaxing the filter specification. Delay caused by filtering and amplification in the error signal path is compensated by adding delay to the IF path. The two paths are then combined in antiphase with a coupler, causing the IMD products to be suppressed at the output of the network.

Although the presence of wanted signal energy in the error path limits system performance, the architecture is simpler than a two loop system, due to the requirement of only a single gain and phase balance adjuster (as compared to the two required in a two-loop system).

This method of using the entire signal at IF frequency appearing at the RF or LO ports as an error signal requires a low wanted signal power relative to that of the IF output at the combination stage. However, in many observed cases, sufficient phase correlation exists between the two wanted signals at the output stage to make this unimportant.

\section{Results}

A demonstrator was built to exploit the signal at the IF frequency appearing at the LO port as an error signal, following the form given in Figure 3. 


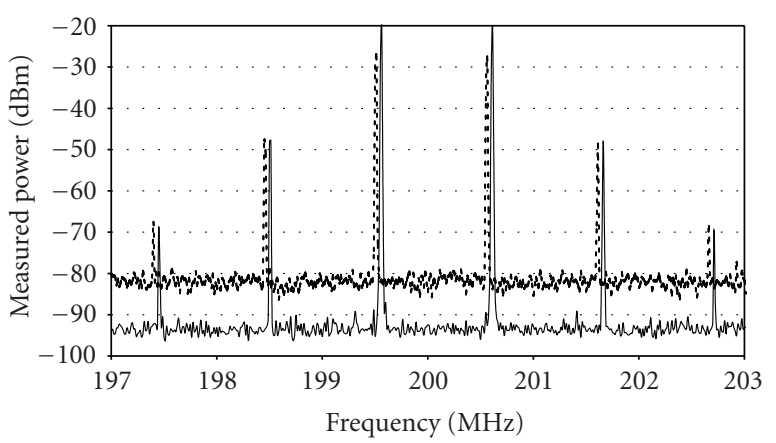

Figure 4: IF band signals entering the output combiner. The hatched trace is the error signal and the solid trace is the IF output signal.

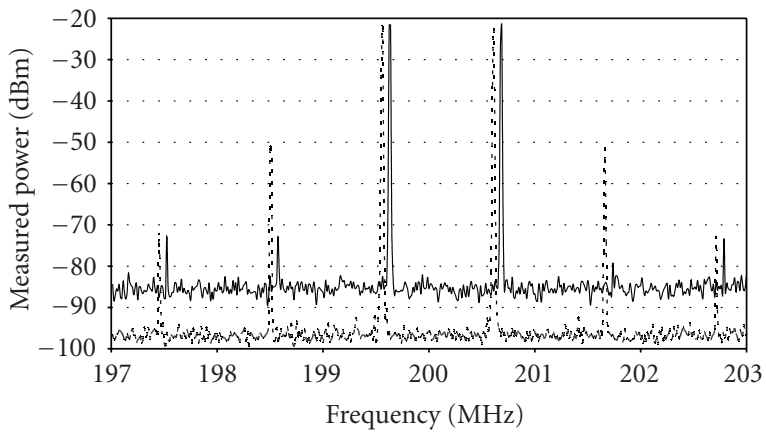

Figure 5: Reduction of mixer distortion. The hatched trace is the system output with the linearization off and the solid trace is the system output with the linearization on.

Figure 4 shows the IF signals appearing at the inputs to the output coupler for a two-tone circuit input. The gain and phase circuit alignments are optimized to linearize the mixer with an input RF of $-7 \mathrm{dBm}$ at 900.55 and $899.50 \mathrm{MHz}$. A $+7 \mathrm{dBm} \mathrm{LO}$ was used at $1.1 \mathrm{GHz}$ (resulting in a $200 \mathrm{MHz} \mathrm{IF}$ ). In Figure 4 a small shift in frequency is applied to one of the traces for clarity. The two signals being combined at the output coupler both contain wanted signal and distortion. The 3IMD is shown to be of equal amplitude in the two paths in Figure 4 both upper 3IMD and lower 3IMD cancel to an extent defined by the amplitude and phase balance accuracy of the system.

4.1. System Performance under Tuned Input Conditions. The result of the combination of the two paths is shown in Figure 5. The 3IMD distortion has been suppressed by $21 \mathrm{~dB}$ (lower) and $28 \mathrm{~dB}$ (upper). The distortion at the 5th-order frequency has been reduced only slightly, due to not being gain/phase balanced in the two combiner paths.

The reduction in wanted signal power may be up to $3.3 \mathrm{~dB}$ with the power difference between the wanted IF signal and the residual wanted signal in the error path of $10 \mathrm{~dB}$ as shown in Figure 1. However, due to the phase correlation as discussed in Section 3, in this case the reduction is negligible.

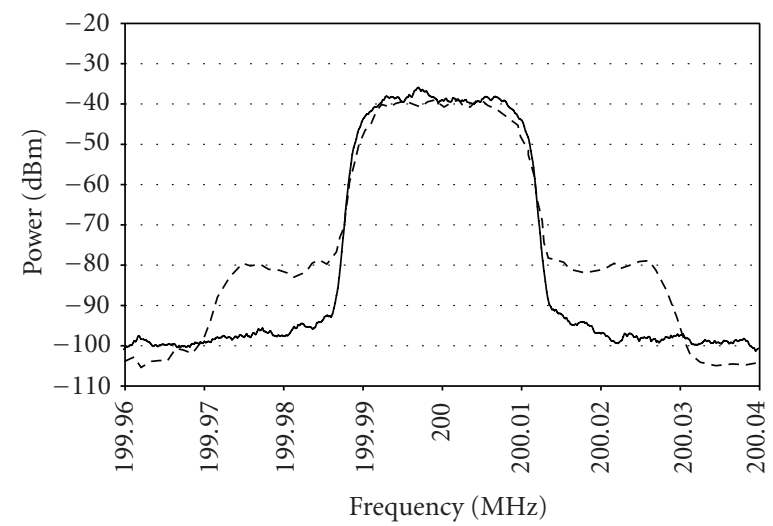

FIgure 6: Linearized frequency translated TETRA signal. The hatched trace is the system output with the linearization off and the solid trace is the system output with the linearization on.

TABLE 1: Performance roll-off under varying input power.

\begin{tabular}{llcl}
\hline $\begin{array}{l}\text { RF input power offset } \\
\text { from onfigured }(\mathrm{dB})\end{array}$ & 2 & 5 & 8 \\
\hline $\begin{array}{l}\text { Suppression reduction } \\
\text { from } 21 \mathrm{~dB}(\mathrm{~dB})\end{array}$ & 18 & 20 & 21 \\
\hline
\end{tabular}

It may be seen in Figure 5 that the majority of the noise power in the error signal is passed to the output of the system. However, due to a limited correlation of this noise to that generated within the mixer and passed to its output port, minor output noise suppression is observed (circa $3 \mathrm{~dB}$ ). The overall increase in SINAD is both noise and distortion limited.

The prototype system was used to demonstrate a reduction in the distortion added to a practical informationbearing signal by the down-conversion operation. Figure 6 shows the result of applying a TETRA signal (Pi/4 DQPSK) to the system. The TETRA signal is of $18 \mathrm{kHz}$ symbol rate with a root Nyquist filter $(\alpha=0.35)$. It is used to modulate a $-7 \mathrm{dBm}$ carrier at $900 \mathrm{MHz}$. A $+7 \mathrm{dBm}$ LO is used at $1.1 \mathrm{GHz}$. This demonstrator system offered around $15 \mathrm{~dB}$ reduction in first adjacent channel power for the TETRAmodulated input.

4.2. Performance under Varying Input Conditions. For maximum processing gain, the 3IMD at the output combiner's two input ports must be equal in power, and in antiphase. This balance is highly sensitive to variation in the input signal. Figure 1 shows that there is a high degree of variance in the 3IMD level difference between IF an LO ports with input power. The suppression performance of the loop is highly sensitive to this variation [6].

Table 1 shows the performance roll-off under varying $\mathrm{RF}$ power level for the demonstrator configured to give the performance shown in Figure 5. The RF input power level is varied but no control was applied to the amplitude and phase control elements. 
TABLE 2: Performance roll-off under varying bandwidth.

\begin{tabular}{lcc}
\hline Bandwidth multiplication factor & 3 & 5 \\
\hline $\begin{array}{l}\text { Suppression reduction from } \\
21 \mathrm{~dB}(\mathrm{~dB})\end{array}$ & 15 & 17 \\
\hline
\end{tabular}

Compared to the configured circuit performance, varying the RF power by as little as $2 \mathrm{~dB}$ causes the circuit to be unbalanced such that any gain given by the linearization scheme is drastically reduced. This observation is supported by the variation in port output power balance with input power seen in Figure 1.

It is feasible to add an active control scheme to counteract the gain and phase variation in 3IMD seen across ports. However, in order to maintain the low difference in 3IMD power across ports as required for this topology, it is better to employ an AGC preamplifier.

Table 2 shows the performance roll-off under varying RF input bandwidth for the demonstrator configured to give the performance shown in Figure 5. The bandwidth of the two-tone input is varied but no control was applied to the amplitude and phase control elements.

With $3 \mathrm{MHz}$ tone separation (multiplication factor of three) the reduction distortion is now $6 \mathrm{~dB}$, compared to the $21 \mathrm{~dB}$ achievable with $1 \mathrm{MHz}$ tone separation. For $5 \mathrm{MHz}$ tone separation (multiplication factor of five) there is a $4 \mathrm{~dB}$ reduction in the distortion at the 3 rd order frequency.

In the varying bandwidth case, it is feasible to add an active control scheme to maintain the optimum balance of amplitude and phase at the output coupler. However, for a fixed-standard receiver topology, the bandwidth requirement is known and so the system can be optimized for a particular bandwidth and input power supplied by the AGC.

\section{Conclusion}

A system has been demonstrated that reduces the near-band distortion products produced by a double balanced diode mixer and the results of a practical investigation is presented. The system uses just a single mixer, exploiting the different signal-distortion ratios of the IF signal found at the IF and LO ports of the mixer for the removal of 3IMD products generated within the mixer at the output frequency. A $21 \mathrm{~dB}$ reduction in the 3IMD products in a $1 \mathrm{MHz}$ bandwidth two-tone-test has been shown and a $15 \mathrm{~dB}$ reduction in the adjacent channel power in a down converting mixer system carrying a TETRA signal has been shown. This linearity enhancement comes at the expense of precise gain and delay balancing. This design restriction is common in suppression loop systems.

Variations in the RF input band, and tone separation are shown to have an adverse affect on the circuit performance; brought about by the abrupt shift in the nonlinear characteristics and the interport output power ratios of these nonlinear signals. Therefore, this approach to mixer linearization is best used when the input power and bandwidth are consistent.

\section{References}

[1] T. Nesimoglu, M. A. Beach, P. A. Warr, and J. R. MacLeod, "Linearised mixer using frequency retranslation," Electronics Letters, vol. 37, no. 25, pp. 1493-1494, 2001.

[2] M. Chongcheawchamnan and I. D. Robertson, "Linearized microwave mixer using simplified feedforward technique," Electronics Letters, vol. 35, no. 9, pp. 724-725, 1999.

[3] P. A. Warr, M. A. Beach, and J. P. McGeehan, "Amplifier linearisation by exploitation of backwards-travelling signals," Electronics Letters, vol. 38, no. 6, pp. 260-261, 2002.

[4] A. Potter, P. A. Warr, and J. P. McGeehan, "An efficient mixer linearisation scheme exploiting inherent error signals," Electronics Letters, vol. 40, no. 20, pp. 1238-1239, 2004.

[5] "Mini-Circuits TUF-5sm Datasheet," http://www.mini-circuits.com/pdfs/TUF-5SM+.pdf.

[6] K. J. Parsons and P. B. Kenington, "Effect of delay mismatch on a feedforward amplifier," IEE Proceedings on Circuits, Devices and Systems, vol. 141, no. 2, pp. 140-144, 1994. 

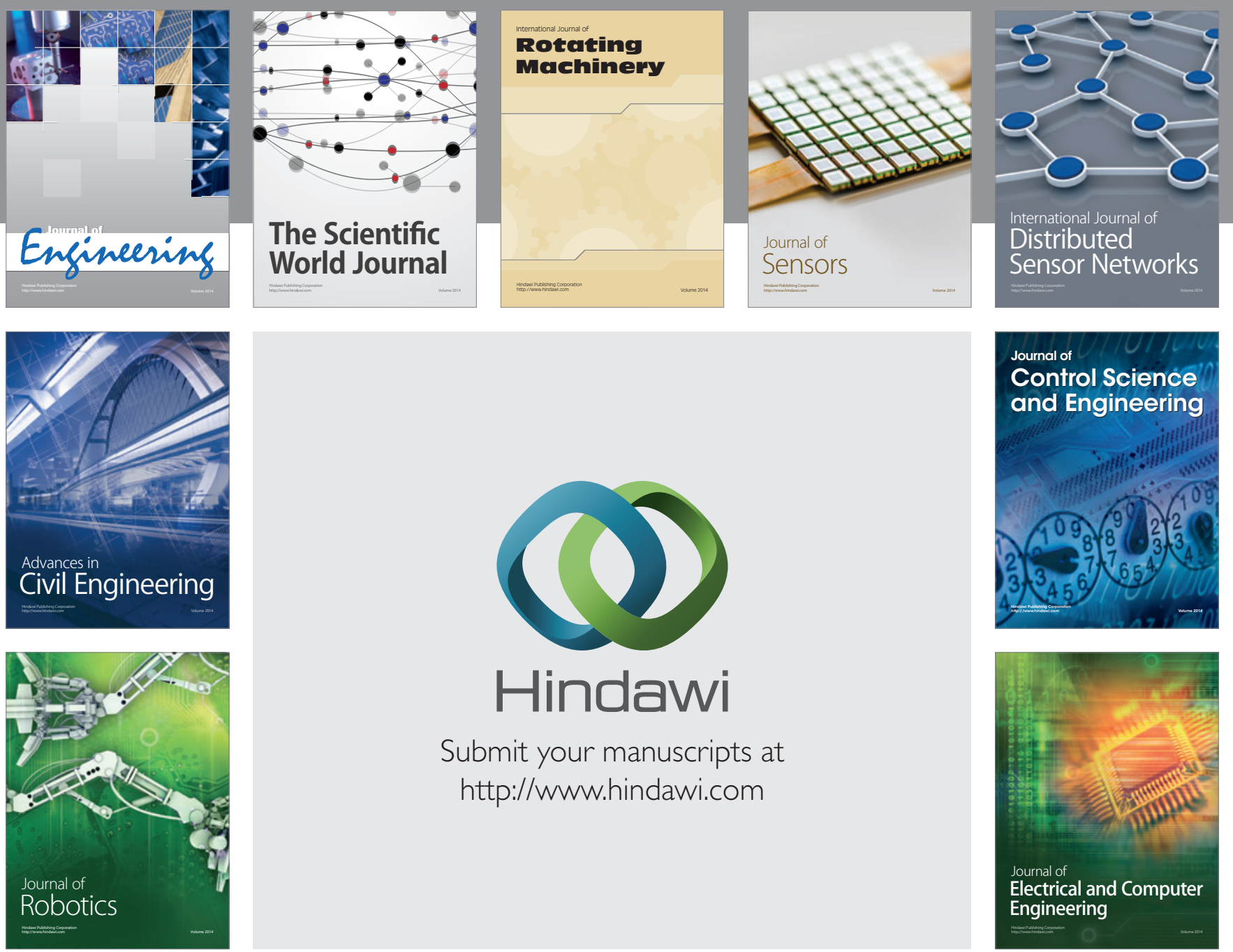

Submit your manuscripts at

http://www.hindawi.com
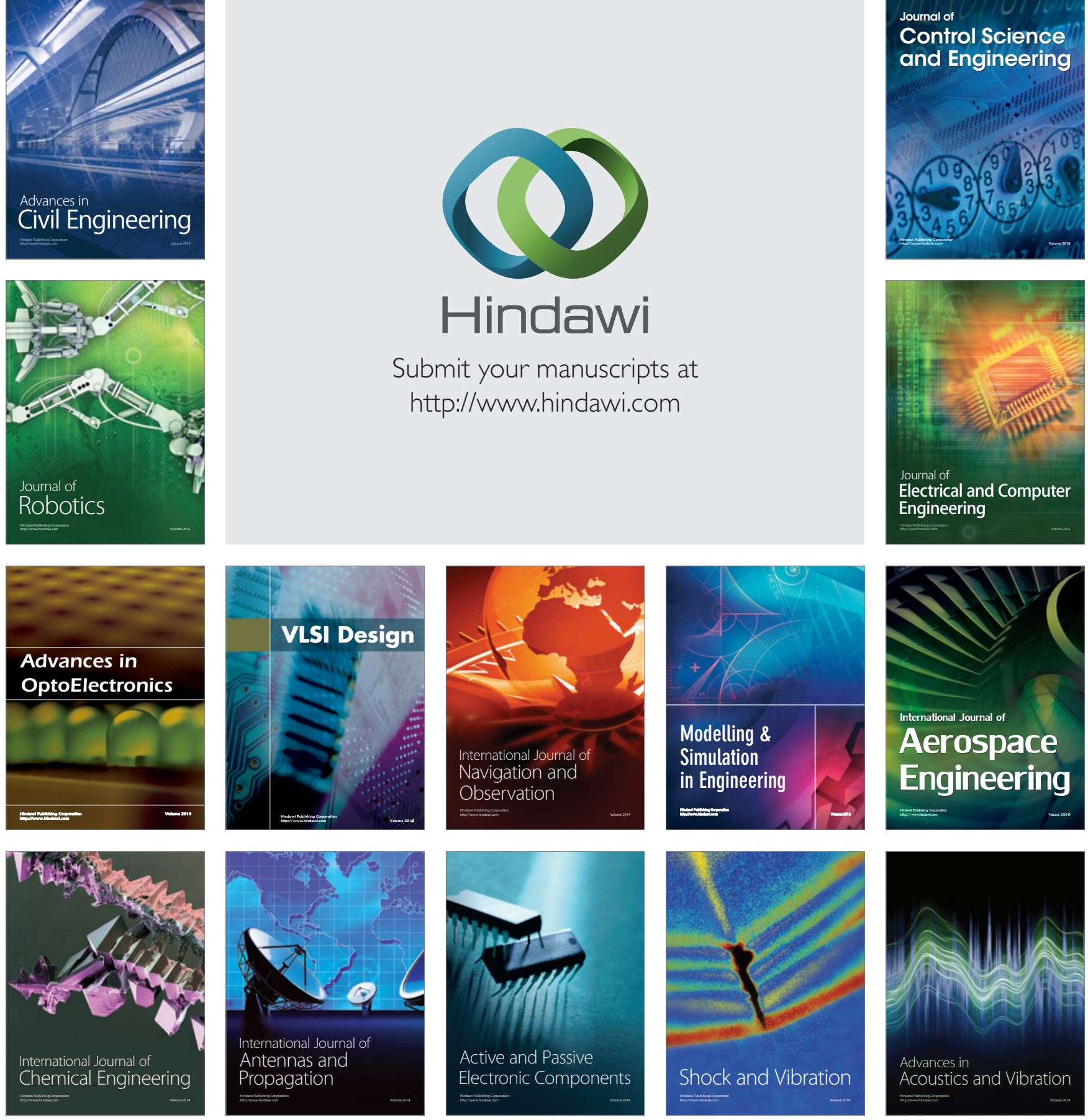